\title{
Ein innovatives Systemmodell für Akutspitäler
}

\author{
Daniel Liedtke ${ }^{a}$, Ole Wiesinger ${ }^{b}$, Christian Westerhoff ${ }^{c}$, Urs Stoffel ${ }^{d}$, Reto Stocker ${ }^{e}$, Stephan Pahls ${ }^{f}$ \\ ${ }^{a}$ Dr., COO Privatklinikgruppe Hirslanden; ${ }^{b}$ Dr. med., CEO Privatklinikgruppe Hirslanden; ${ }^{c}$ Dr. med., CCO Privatklinikgruppe Hirslanden; ${ }^{d}$ Dr. med., Chirurgische
} Praxis Enge, Zürich; ${ }^{e}$ Prof. Dr. med., Institut für Anästhesiologie und Intensivmedizin Klinik Hirslanden, Zürich; ${ }^{\dagger}$ Dr. med., Pahls Consulting, Zürich

\begin{abstract}
Als Folge der neuen Spitalplanung und -finanzierung befindet sich die Spitallandschaft der Schweiz in einem tiefgreifenden Wandel. Viele Spitäler begegnen den Herausforderungen aktiv, mit Massnahmen wie Umwandlung von Rechtsformen, Lean Management und Erneuerung der Spitalinfrastruktur. Die Organisation der Spitalärzteschaft wird aber noch zu wenig als Erfolgsfaktor wahrgenommen.
\end{abstract}

Als Folge der neuen Spitalplanung und -finanzierung befindet sich die Spitallandschaft der Schweiz in einem tiefgreifenden Wandel. Die Kantone schreiben den Spitälern im Detail vor, welche Leistungen sie erbringen dürfen, welche Qualitätsanforderungen zu erfüllen sind, wie viel Personal auszubilden ist und welche Fallkosten als akzeptabel gelten. Untereinander stehen die Spitäler im Wettbewerb um Patienten, Pflegepersonal und die besten Ärzte, sind aber gleichzeitig durch Fachkräftemangel, Kostendruck und Spitallisten im Handlungsspielraum eingeschränkt. Viele Spitäler reagieren auf diese Herausforderungen mit der Umwandlung der Rechtsform, Lean Management, Erneuerung der Spitalinfrastruktur, Bildung von Kompetenzzentren und verstärkten Kooperationen. Ist das genug? Unseres Erachtens werden zwei wichtige Themen vernachlässigt: die Organisation der Ärzteschaft und die Zuteilung von Aufgaben an die Ärzteschaft. Diese Themen sind zentral für alle Spitäler und beeinflussen ihre Wettbewerbsfähigkeit und den Patientennutzen entscheidend und nachhaltig.

\section{Zurück zu sinnvoller Arbeitsteilung}

Zum einen geht es um die Rückbesinnung auf eine sinnvolle Arbeitsteilung im Spital. Die nicht-klinischen Aufgaben des ärztlichen Kaders sind in den letzten Jahren stetig angestiegen. Dies schmälert die Attraktivität des Arbeitsplatzes, vermindert die Zeit für Patientenkontakte, ist nicht kosteneffizient und angesichts des Fachärztemangels langfristig nicht tragbar. Zum anderen geht es um eine funktional zweckmässige Organisation der Ärzteschaft im Spital. In den letzten Jahren kristallisieren sich zwei unterschiedliche spitalärztliche Tätigkeitsprofile heraus. Auf der einen Seite ste- hen die ärztlichen Querschnittfunktionen wie z.B. Anästhesie und Intensivmedizin, Allgemeine Innere und Notfallmedizin, oder auch die Radiologie, die als ärztliche Dienstleister oder "Grundversorger» im Spital agieren sollen. Auf der anderen Seite stehen stetig stärker sub- oder «super»-spezialisierte ärztliche Teams, die auf spezifische Bedürfnisse von Zuweisern und Patienten einzugehen haben. Es zeigt sich, dass diese unterschiedlichen ärztlichen Aufgaben auch unterschiedliche Organisation und Führung erfordern.

Die Organisation der Ärzteschaft und die Zuteilung von Aufgaben an die Ärzteschaft sind zentral.

Obwohl Porter und Teisberg schon vor bald 10 Jahren ihr Modell der Integrated practice units (IPU) beschrieben haben [1], verharrt die Organisation der Ärzteschaft in vielen Spitälern immer noch in traditionellen Mustern. Wie werden Spitäler und Ärzte ihr Verhältnis weiterentwickeln? Dazu wurde der Zürcher Wirtschaftsprofessor Tilman Slembeck kürzlich im SonntagsBlick mit einer bemerkenswerten These zitiert [2]. Laut Slembeck werden Spitäler künftig vor allem zu Hardwarezentren, die Apparate, Untersuchungs- und Operationsräume zur Verfügung stellen. Operiert werde ähnlich wie beim Belegarztsystem von frei praktizierenden Medizinern, die sich in Ärztenetzwerken organisieren. Festangestellte Chirurgen solle es demnach in 10 bis 15 Jahren fast nur noch in Unispitälern geben. Diese These ist gewagt, aber nicht ganz abwegig, denn vergleichbare Shop-in-shop-Lösungen kennen wir auch aus anderen Bereichen in der Wirtschaft. Wir möchten die These aufnehmen und am Beispiel der Klinik Hirslanden in Zürich zeigen, dass eine funk- 
tionsspezifisch differenzierte Organisation der Ärzteschaft handfeste Vorteile für Patienten, Ärzte, Spitäler und Kostenträger bietet.

\section{Das Hirslanden-System: Fusion des Chefarzt- und Belegarztsystems}

In den letzten 10 Jahren haben Spitalleitung und Ärzteschaft die Klinik Hirslanden vom reinen Infrastrukturanbieter für mehrheitlich solitäre Belegärzte zu einem umfassenden stationären Systemanbieter mit organisierten Belegarztzentren transformiert, so dass die Klinik Hirslanden heute als Listenspital das Leistungsspektrum eines Maximalversorgers anbieten kann. Ein wesentlicher Erfolgsfaktor war dabei die differenzierte Organisation der Ärzteschaft im sogenannten "Hirslanden-System», das eine Fusion des Chefarzt- und Belegarztsystems darstellt. Dieses System definiert die Rolle und Organisation der Ärzte unterschiedlich für medizinische Basisleistungen und für spezialisierte Leistungen - und bildet damit in gewissem Sinne das Leistungsgruppenkonzept der Zürcher Spitalplanung ab.

Das Basispaket der Spitalliste beinhaltet mit der Notfallmedizin, Anästhesie, Intensivmedizin, Allgemeinmedizin, Allgemeinchirurgie und Radiologie eine Art stationäre Grundversorgung. Der Begriff «Grundversorgung» oder "Basisversorgung» sollte dabei nicht falsch verstanden werden. Er bezeichnet die Funktion einer Leistung im Gesamtspital, nicht den Komplexitätsgrad dieser Leistung. Das heisst, aus fachlicher Sicht als (hoch-)spezialisiert zu bezeichnende Leistungen der Intensivmedizin werden aus funktionaler Sicht dennoch der spitalinternen Grundversorgung zugeordnet.

Im Hirslanden-Modell sind die ärztlichen und nichtärztlichen Dienste der spitalinternen Grundversorgung in der Organisationseinheit «Medizinisches System» zusammengefasst, das von einem Arzt ohne klinische Aufgaben in der Funktion eines vollamtlichen Managers der medizinischen Kernprozesse geführt wird. Die Ärzte des Medizinischen Systems werden im Chefarzt-System geführt und sind wie die Pflegenden und Therapeuten vom Spital angestellt. Das Medizinische System strebt eine hohe Integration seiner ärztlichen und nichtärztlichen Dienste und damit eine hohe interdisziplinäre Integration der Behandlungsprozesse an.

Demgegenüber stehen ärztliche Zentren (Herzzentrum, Fusszentrum usw.), die in Zusammenarbeit mit dem Medizinischen System spezialisierte und hochspezialisierte Leistungen erbringen. Die Zentren sind meist als monodisziplinäre, in den Spitalräumlich- keiten eingemietete, kollegial geführte, belegärztliche Gruppenpraxen mit unterschiedlichen Rechts- und Organisationsformen (z.B. Praxisgemeinschaft, Partnermodell analog Anwaltskanzleien oder Aktiengesellschaft mit angestellten Ärzten) aufgestellt. Die Inhouse-Zentren sind durch transparente Verträge und eine ausgereifte Prozessorganisation eng in den Spitalbetrieb eingebunden und u.a. verpflichtet, am Notfalldienst des Fachgebiets teilzunehmen, die Qualitätsstandards des Spitals einzuhalten und zentrumsintern einheitliche Behandlungsstandards festzulegen.

Die Ärzte des Medizinischen Systems stellen also ihre Dienstleistung den Patienten und Zentrumsärzten im Sinne einer integrierten Grundversorgung zur Verfügung, managen Co-Morbiditäten und Komplikationen und erlauben so dem Zentrumsarzt, sich auf seine spezialisierte Kerntätigkeit zu konzentrieren, während der Zentrumsarzt durch guten Outcome und Zuweiserpflege kontinuierlich die Marktposition seines Zentrums behaupten muss. Gesteuert wird die so gegliederte Leistungserbringung durch die direkt der Spitalleitung unterstellte zentrale OP- und Bettendisposition und das spitalübergreifende klinische Qualitätsmanagement (Koordination von Behandlungsstandards, Indikationsboards, M\&M-Boards, CIRS Registerführung, Spitalhygiene usw.).

Ein Arzt ohne klinische Aufgaben betreut die Einheit "Medizinisches System» als Manager medizinischer Kernprozesse.

Diese duale Organisation der Ärzteschaft (ärztliche Grundversorger im Chefarzt-System und spezialisierte belegärztliche Zentren), ergänzt durch ein zentrales Qualitäts- und Performance-Management, hat sich für die Ärzteschaft und die Leitung der Klinik Hirslanden so gut bewährt, dass das Modell zum Vorbild für die ganze Hirslanden-Gruppe geworden ist.

\section{Die Grundelemente des Systemmodells für Akutspitäler}

Weil wir überzeugt sind, dass dieses Modell auch anderen Spitälern unabhängig von Rechtsform oder Trägerschaft helfen kann, den Herausforderungen der Zukunft zu begegnen und insbesondere den Patientennutzen zu erhöhen, möchten wir ein adaptiertes Systemmodell mit den folgenden vier Grundelementen zur Diskussion stellen:

\section{Medizinische Service-Einheiten}

Die Medizinischen Service-Einheiten (Leistungserbringung; in Abb. 1 grün markiert) sind Grundver- 
sorger im Spital und Dienstleister für Patienten und Spezialisten. Sie umfassen ärztliche (Anästhesie, Intensivmedizin, Radiologie, Notfallmedizin u.a.) und nichtärztliche Service-Einheiten (Pflege, Therapien u.a.) und sind einer gemeinsamen Leitung unterstellt. Sie betreiben die zentrale medizinische Spitalinfrastruktur wie den Notfall, den OP, die Intensivstation und die Bettenstationen und stellen die Notfallbereitschaft in der Grundversorgung sicher. Ihr primäres gemeinsames Ziel ist eine hohe interdisziplinäre Integration aller stationären Behandlungsprozesse und die Patientensicherheit. In enger Abstimmung mit den Spezialisten-Einheiten sind die Service-Einheiten in alle Prozessschritte der Patientenbehandlung von Spitaleintritt bis -austritt involviert. Die Ärzte der Service-Einheiten sind vom Spital angestellt und werden im Chefarzt-System geführt. Diese Chefärzte sind direkt dem gemeinsamen Leiter der Medizinischen Service-Einheiten unterstellt, welcher Mitglied der Spitalleitung sein sollte.

\section{Medizinische Spezialisten-Einheiten}

Die Medizinischen Spezialisten-Einheiten (Leistungserbringung; in Abb. 1 dunkelblau markiert) auf Stufe der Subspezialitäten (z.B. Gastroenterologie, Wirbelsäulenchirurgie) tragen unternehmerische Verantwortung für die Erbringung von spezialisierten medizinischen Leistungen, Zuweiserpflege, Patientenakquise und Innovation im Fachgebiet und stellen in Kooperation mit den Service-Einheiten die Notfallbereitschaft für das jeweilige Fachgebiet sicher. Die Ärzte der Spezialisten-Einheiten sind vom Spital angestellt oder Belegärzte und können verschiedenartig organisiert sein (z.B. Chairman-System, Partner-System oder auch Chefarzt-System), wobei eine partnerschaftlich-kollegiale Ausrichtung zu gewährleisten ist.

\section{Medizinisches Qualitäts- und Performance- Management}

Das Medizinische Qualitäts- und Performance-Management (Ordnungseinheit zur Steuerung der Leistungserbringung; in Abb. 1 hellblau markiert) sichert durch zentrale OP- und Bettenplanung und zentrales klinisches Qualitätsmanagement spitalweit Qualität und Wirtschaftlichkeit und entlastet damit die medizinischen Service-Einheiten und Spezialisten-Einheiten von planerischen und administrativen Aufgaben. Mit dem Ziel einer effektiven und interessenunabhängigen

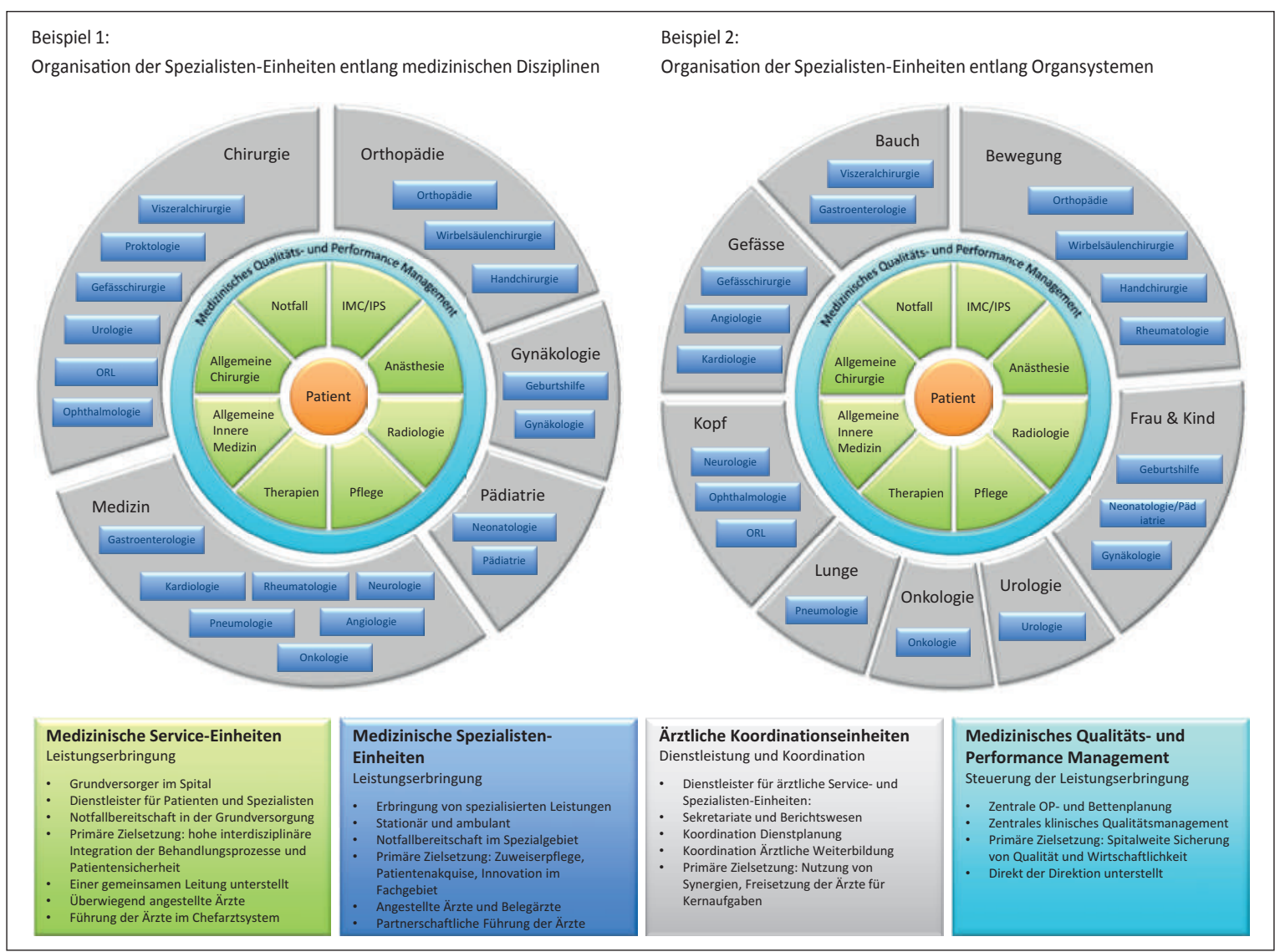

Abbildung 1: Möglichkeiten der Ausgestaltung des Systemmodells in Akutspitälern (Auswahl der Spezialisten-Einheiten nur beispielhaft). 
Gewaltenteilung sollte diese Einheit nicht von leitenden Kadern der Service-Einheiten oder SpezialistenEinheiten geführt werden, sondern ärztlich geleitet direkt der Spitaldirektion unterstellt werden.

\section{4. Ärztliche Koordinationseinheiten}

Die Ärztlichen Koordinationseinheiten (Dienstleistungseinheiten; in Abb. 1 grau markiert) übernehmen koordinative und administrative Aufgaben wie Dienstplanung, Berichtswesen, Organisation der ärztlichen Weiterbildung, Koordination von Behandlungsstandards zwischen Fachspezialitäten usw. für eine Gruppe von fachlich verwandten ärztlichen Service- und Spezialisten-Einheiten. Sie nutzen dadurch Synergien und generieren im nicht-klinischen Bereich Effizienzgewinne, die mehr Raum für ärztliche Kernaufgaben schaffen

Gegenüber den heute in vielen Spitälern vorherrschenden Managementsystemen legt unser Modell stärkeres Gewicht auf Arbeitsteilung zwischen Professionen und Betriebseinheiten, sowie eine funktionsspezifisch angepasste innere Organisation der Betriebseinheiten und der ärztlichen Führung.

Angesichts der vielfältigen Rahmenbedingungen der Schweizer Spitäler beschränkt sich unser Modell bewusst auf einige wenige Prinzipien. Die organisatorische, führungstechnische und vertragliche Umsetzung wird an verschiedenen Orten unterschiedlich aussehen und auch die sinnvolle Umsetzungstiefe kann variieren. Selbst die Privatklinikgruppe Hirslanden implementiert nicht alle Elemente des Modells in allen ihren Kliniken. Dennoch sind wir überzeugt, dass die beschriebenen Modellelemente eine gewisse Allgemeingültigkeit und grosses Potential für das moderne Krankenhausmanagement haben.

In diesem Sinne ist das Beispiel in Abbildung 1 als ideal-typische, schematische Veranschaulichung zu verstehen, die für jedes Spital individuell anzupassen ist.

\section{Das Systemmodell schafft Potentiale für die Ärzteschaft}

Das Modell schafft insbesondere für die Ärzteschaft bedeutende Potentiale:

- Für Kaderärzte generell: Effizienzgewinne in nichtklinischen Aufgaben und dadurch Zeitgewinn für ärztliche Kernaufgaben.
- Steigerung von Patientensicherheit und -nutzen, Servicequalität und Prozesseffizienz durch stärkere Integration und einheitliche Führung der medizinischen Grundversorgung in den Service-Einheiten.

- Spitalweit zentralisierte und dadurch effizientere Ressourcenbewirtschaftung (OP-Planung, Bettendisposition).

- Spitalweit zentralisiertes und dadurch zuverlässiger $\mathrm{zu}$ steuerndes und $\mathrm{zu}$ verbesserndes klinisches Qualitätsmanagement.

- Nutzung des unternehmerischen Potentials von Spezialisten in den Spezialisten-Einheiten.

- Erleichterte Einbindung von Belegärzten in allen Subspezialitäten (nicht nur in kleinen Fächern).

- Erleichterung von Teilzeitarbeit unter Spezialisten, sowie Arbeit von Spezialisten im Teilzeitpensum in mehreren Spitälern und dadurch Entschärfung des Fachärztemangels.

- Steigerung der Attraktivität des Spitals für hochqualifizierte Spezialisten aufgrund von flexiblen Beschäftigungslösungen und mehr unternehmerischer Freiheit.

- Überbrückung der Distanz zwischen primär im stationären Bereich tätigen angestellten Spitalärzten und primär im ambulanten Bereich tätigen freischaffenden Spezialärzten - dadurch erweist sich das Modell als zeitgemässe Antwort auf die anhaltende Verschiebung von stationären Leistungen in den ambulanten Bereich.

Das Modell erfordert Umdenken. Die klassische, hierarchisch organisierte ärztliche «Klinik» soll zu einer schlanken Koordinationseinheit ohne Planungshoheit über Betten oder OP-Kapazitäten werden. Die Funktion "Chefarzt» muss neu ausgerichtet werden: in erster Linie auf die ärztlichen Kernaufgaben von klinischer Arbeit, ärztlicher Weiterbildung und Innovation im Fachgebiet. Belegärzte müssen sich noch stärker zu kollegial organisierten Spezialisten-Einheiten formieren, die eng mit den Service-Einheiten kooperieren. Das ist nicht von heute auf morgen zu erreichen, aber äusserst lohnenswert.

Ausgewählte Elemente und Funktionen des Systemmodells werden wir in einem Folgeartikel in der nächsten Ausgabe der Schweizerischen Ärztezeitung vorstellen.

Literatur

1 Michael E. Porter, Elizabeth O. Teisberg. Redefining Health Care: Creating Value-Based Competition on Results. 2006.

2 Spitäler verlochen Milliarden. SonntagsBlick vom 22. Februar 2015

- Weitere Literatur beim Erstautor. 